Historic, archived document

Do not assume content reflects current scientific knowledge, policies, or practices. 


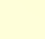


UNITED STATES DEPARTMENT OF AGRICULTURE AGRICULTURAL MARKETING SERVICE FRUIT AND VEGETABLE DIVISION

WASHING TON $28, D$. C.

\section{UNITED STATES STANDARDS FOR GRADES OF PROCESSED FRUITS, VEGETABLES, AND CERTAIN OTHER PRODUCTS}

(As of July 2, 1959)

CANNED (and similarly processed) I/

Apples

Apple Butter

Apple Juice

Applesauce

Apricots

Asparagus

Beans, Dried

Beans, Green and Wax

Beans, Lima

Beets

Blackberries and other similar berries

Blueberries

Carrots

Cherries, Red Sour (Tart) Pitted

Cherries, Sweet

Chili Sauce

Corn, Cream Style

Corn, Whole Kernel (or Whole Grain)

Cranberry Sauce

Figs, Kadota

Fruit Cocktail

Fruit Jelly

Fruit Preserves (or Jams)

Fruits for Salad

Grapefruit

Grapefruit Juice

Grapefruit Juice for Manufacturing

Grapefruit and Orange Juice, Blended

Grapefruit and Orange for Salad

Grape Juice
EFFECTIVE

October 17, 1953

August 5, 1957

June 26, 1950

September 18, 1950

May 17, 1957

March 12, 1957

October 24, 1947

March 2, 1953

July 21, 1953

February 4, 1955

December 1, 1956

March 20, 1951

Ju1y 2, 1959

June 23, 1949

February 25, 1957

October 20, 1953

July 27, 1953

July 30, 1952

March 19, 1951

August 16, 1957

August 17, 1953

January 2, 1948

May 25, 1956

April 1, 1942

January 19, 1954

October 19, 1954

March 20, 1957

October 19, 1954

August 7, 1950

May 14, 1951

1) In all standards in these groups, effective prior to July 1, 1957, the "Tolerances for Certification of officially Drawn Samples" were deleted by blanket amendment in the revised "Regulations Governing Inspection and Certification of Processed Fruits and Vegetables and Related Products," effective July 1, 1957. The new procedure for ascertaining the grade of a lot based on officially drawn samples is covered in sections 52.13 and 52.38 of the revised regulations. 
CANNED (and similarly processed) I/

$$
\text { - continuation }
$$

Hominy

Lemon Juice

Mushrooms

Okra

Olives, Green

Olives, Ripe

Onions

Orange Juice

Orange Juice, Concentrated

Orange Juice, Concentrated for Manufacturing

Orange Marmalade

Peaches, Freestone

Peaches, Yellow Clingstone

Pears

Peas

Peas, Field and Black-eye

Pickles, Cucumber

Pimientos

Pineapple

Pineapple Juice

Plums

Potatoes, White

Prunes, Canned Dried

Pumpkin (Squash)

Raspberries, Red

Sauerkraut

Spinach

Squash (Summer Type)

Succotash

Sweetpotatoes

Tangerine Juice

Tangerine Juice, Concentrated for

Manufacturing

Tomatoes

Tomato Catsup

Tomato Juice

Tomato Paste

Tomato Pulp

Tomato Sauce

Tomatoes and Okra
EFFECTIVE

March 10, 1958

July I, 1941

January 19, 1953

July 8, 1957

June I, 1946

March 1, 1941

November 2, 1957

March 13, 1956

December 12, 1953

December 20, 1957

June 22, 1951

July 22, 1957

July 13, 1957

December I, 1951

May 13, 1955

April 17, 1950

April 30, 1954

August 16, 1955

March 16, 1957

March 16, 1957

August 19, 1952

February 10, 1950

March 1, 1946

March 9, 1956

March 1, 1958

May 10, 1957

May 12, 1950

May 25, 1959

March 15, 1944

July 9, 1951

July 29, 1949

October 31, 1955

August 1, 1946

August 31, 1953

June 24, 1958

September 15, 1944

January 1, 1945

May 10, 1954

December 24, 1957 
DRIFD OF DEHYDRATED (IOH-moisture)

(If so stated)

Apples

Apples, Dehydrated (Iow-moisture) 2/

Apricots

Currants

Dates 2/

Figs

Grapefruit Juice (Dehydrated) 2/

Orange Juice (Dehydrated) 2/

Peaches

Pears

Prunes

Raisins, Processed

FROZFN or CHILLED (If so stated) If

Apples

Apricots

Asparagus

Grades for Manufacturing

Beans, Green and Wax

Beans, Lima

Berries, (Blackberries, Boysenberries, etc.)

Blueberries

Broccoli

Brussels Sprouts

Carrots, Diced

Cauliflower

Cherries, Red Sour (Tart) Pitted

Cherries, Sweet

Corn-on-the-Cob

Corn, Whole Kernel (or Whole Grain)

Grapefruit

Grapefruit Juice, Concentrated

Grapefruit Juice and Orange Juice,

Concentrated, Blended

Grape Juice, Concentrated Sweetened

Leafy Greens

27 The "Tolerances for Certification of Officiajiy Drawn Samples" in the standards for these products were deleted by blanket amendment in the revised "Regulations Governing Inspection and Certification of Processed Fruits and Vegetables and Related Products," effective July 1, 1957. The new procedure for ascertaining the grade of a lot based on officially drawn samples is covered in sections 52.13 and 52.38 of the revised regulations.
October 24, 1955

November 29, 1955

June I, 1945

October 1, 1957

August 26, 1955

December 27, 1955

March 19, 1956

January 27, 1956

June 30, 1945

June 30, 1945

November 26, 1956

October 1, 1957

May 17, 1954

June 30, 1945

Juzy I, 1946

April 18, 1952

August 25, 1954

April 16, 1957

August 15, 1946

May 7, 1955

March 1, 1959

May 11, 1951

March 30, 1953

November 12, 1951

June 18, 1949

March 1, 1958

May 15, 1958

August 1, 1952

February 20, 1948

December 28, 1956

December 10, 1951

November 1, 1957

June 13, 1952 


\begin{tabular}{|c|c|}
\hline $\begin{array}{c}\text { FROZEN or CHIIIEE (If so stated) I] } \\
\text { - continuation }\end{array}$ & EFFECTIVE \\
\hline $\begin{array}{l}\text { Lemonade, Frozen Concentrate } \\
\text { limeade, Frozen Concentrate } \\
\text { Okra } \\
\text { Orange Juice, Chilled } \\
\text { Orange Juice, Concentrated } \\
\text { Peaches } \\
\text { Peas } \\
\text { Peas and Carrots } \\
\text { Peas, Field and Black-eye } \\
\text { Feppers, Sweet } \\
\text { Pineapple } \\
\text { Plums } \\
\text { Potatoes, French Fried } \\
\text { Raspberries } \\
\text { Rhubarb } \\
\text { Spinach } \\
\text { Squash (Cooked) } \\
\text { Squash (Sumer Type) } \\
\text { Strawberries } \\
\text { Succotash } \\
\text { Turnip Greens with Turnips } \\
\text { Vegetables, Mixed }\end{array}$ & 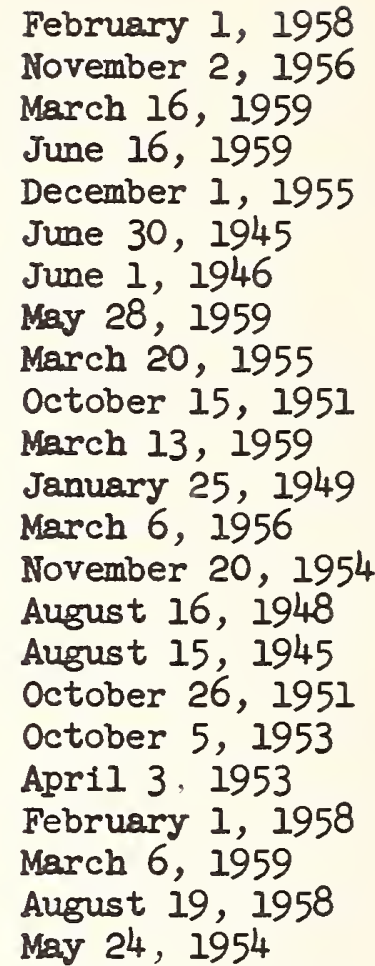 \\
\hline
\end{tabular}

SUGAR PRODUCTS

Honey, Comb

Honey, Extracted 2/

Maple Sirup for Reprocessing

Maple Sirup, Table

Molasses, Sugarcane 2/

Sirup, Refiners' $2 /$

Sirup, Sugarcane $2 /$

August 1933

April 16, 1951

February 15, 1940

February 15, 1940

August 11, 1952

January 5, 1952

Apri1 16, 1957

MISCELIANEOUS PRODUCTS

Cherries, Sulfured 2/

Olive Oil

Olives, Salt Cured

Olives, Sicilian Style

Peanut Butter 2/

Potatoes, Peeled White (Fresh) $2 /$

June 12, 1951

March 22, 1948

November 25, 1940

November 25, 1940

September 1, 1942

June 8,1954

Sauerkraut, Bulk

May 27, 1935

Any of the United States Standards for Grades listed may be secured upon

request from: Processed Products Standardization

and Inspection Branch

Fruit and Vegetable Division, AMS

U. S. Department of Agriculture

Washington 25, D. C. 

